\title{
Modern Methodological Approaches to Rehabilitation of Disabled Patients from Ischemic Heart Disease
}

\section{Bodrova Reseda $\mathrm{A}^{1 *}$, Iksanov Haydar $\mathrm{V}^{1}$, Ishteryakova Olga $\mathrm{A}^{2}$ and Iunusova Endzhe $\mathbf{R}^{3}$}

${ }^{1}$ Kazan State Medical Academy - Branch of the Russian Medical Academy of Continuing Professional Education of the Ministry of Health of the Russian Federation, Kazan, Republic of Tatarstan, Russian Federation

${ }^{2}$ Kazan State Medical University of the Ministry of Health of the Russian Federation, Kazan, Republic of Tatarstan, Russian Federation

${ }^{3}$ City Clinical Hospital № 7, Kazan, Republic of Tatarstan, Russian Federation

*Corresponding Author: Bodrova Reseda A, Kazan State Medical Academy Branch of the Russian Medical Academy of Continuing Professional Education of the Ministry of Health of the Russian Federation, Kazan, Republic of Tatarstan, Russian Federation.
Received: May 08, 2021

Published: July 26, 2021

(C) All rights are reserved by Bodrova

Reseda A., et al.

\section{Abstract}

The article makes a detail review of goals, principles and stages of medical and social rehabilitation of people with disabilities due to coronary heart disease. The list, content, and level of rehabilitation measures carried out within the framework of an individual program of rehabilitation or habilitation of a disabled person are shown. Here is indicated the specific testing methods by using functional diagnostic tests that simulate various types of loads in life. The most important principles for assessing the rehabilitation potential and determining the rehabilitation prognosis had formulated.

Keywords: Ischemic Heart Disease; Individual Rehabilitation or Habilitation Program for the Disabled; Rehabilitation Prognosis; Rehabilitation; International Classification of Functioning; Limitation of Life and Health

\section{Introduction}

In the structure of disability among diseases of the cardiovascular system, ischemic heart disease (hereinafter - IHD) occupies a leading place. Rehabilitation tactics in relation to a patient with coronary artery disease with coronary deficiency depends on the results of the analysis of the survey. A large number of examination methods are currently used to diagnose the condition of patients, in addition, the number of specific methods of exposure has increased. As a result, it is difficult for a specialist choosing a rehabilitation tactics to collect and analyze the information necessary for making a decision [4].
This can lead to the choice of the wrong tactics and a decrease in the effectiveness of rehabilitation. The coordinated model, created on the basis of high technologies, will make it possible to unify the patient's rehabilitation system and quickly select the optimal list of rehabilitation measures for everyone.

Medical and social rehabilitation of disabled people due to IHD is carried out in accordance with individual programs of rehabilitation or habilitation of disabled people.

Medical and social rehabilitation of disabled due to IHD

The main goal of medical and social rehabilitation of people with disabilities due to coronary artery disease is their integration 
into society. At the same time, according to the developed concept, medical and social rehabilitation of this contingent of disabled people based on the following principles:

- Guarantee of observance of rights in the field of medical, professional and social rehabilitation at the state level

- $\quad$ Priority of the interests of disabled people in the implementation of rehabilitation measures

- General availability of the rehabilitation system based on taking into account the physical, psychophysiological and social characteristics of people with disabilities due to IHD

- The principle of non-causality, according to which all types of necessary rehabilitation assistance must be provided regardless of the cause of the disability

- The principle of intervention at the earliest possible stage, when, depending on the possibility and need, all measures should be taken to reduce the scale and consequences of disability to a minimum, and the inevitable consequences are compensated in the most effective way

- $\quad$ The principle of providing individual assistance depending on the specific needs of each person with disabilities

- A variety of forms and methods of rehabilitation for ischemic heart disease based on a systematic approach in their implementation

- The state-public nature of the management of the rehabilitation system for the disabled [3].

The implementation of these principles is carried out with the help of rehabilitation programs and state rehabilitation standards of rehabilitation institutions, governing bodies and their subordinate structures involved in the rehabilitation of disabled people. Rehabilitation programs for IHD are based on aspects of medical, professional and social rehabilitation of disabled people [7].

Medical rehabilitation of disabled people due to IHD is a set of measures and types of assistance necessary to treat and prevent the deterioration of the patient's condition, prevent the onset and aggravation of disability.
Medical rehabilitation includes:

- $\quad$ Reconstructive surgery

- $\quad$ Prosthetics and orthotics

- $\quad$ Sanatorium treatment.

Medical rehabilitation for ischemic heart disease involves the use of therapeutic agents, including pharmacotherapy, physiotherapy, kinesitherapy, exercise therapy, massage, occupational therapy. Reconstructive surgery for ischemic heart disease includes the techniques of coronary artery bypass grafting, coronary grafting, etc.

The main forms of medical rehabilitation of disabled people due to ischemic heart disease are stationary, sanatorium-resort and outpatient. The stationary form of medical rehabilitation is used for intensive targeted medical rehabilitation, as a result of which a certain restoration and stabilization of previously impaired functions of organs and systems is achieved. The stationary form of rehabilitation, as a rule, is the basis of the initial form in the system of the so-called staged rehabilitation for ischemic heart disease.

Sanatorium treatment is a type of therapeutic and prophylactic assistance to the population. Based on the predominant use of natural healing factors (climate, mineral waters, therapeutic mud, sea bathing, etc.). It is carried out taking into account the achievements of balneology, the clinic of internal diseases and other medical disciplines. The complex of factors of sanatorium-resort treatment also includes a change in the situation and exclusion of the patient from the usual working and living conditions, the peculiarities of natural conditions and the landscape of resorts, etc. -social rehabilitation of sick and disabled people [5].

For the full development of plans for medical rehabilitation and assessment of rehabilitation measures in patients with IHD, a quantitative characteristic of the degree of impaired functions, activity and participation is required. The most priority tools for assessing quantitative indicators of the effectiveness of medical and social rehabilitation of disabled people due to IHD are the International Classification of Functioning, Disabilities and Health (ICF) and the Rankin scale [6]. 
Rehabilitation of persons with disabilities due to ischemic heart disease is a dynamic process, the main stages of which are: carrying out expert-rehabilitation diagnostics and the development of individual rehabilitation programs on its basis, correction of the content of individual rehabilitation and habilitation programs for persons with disabilities.

Medical and social rehabilitation is carried out on the basis of the regulatory document "On approval of the procedure for the development and implementation of an individual rehabilitation or habilitation program for a disabled person, an individual rehabilitation or habilitation program for a disabled child issued by federal state institutions of medical and social expertise, the procedure for their development and implementation", approved by order Ministry of Labor and Social Protection of the Russian Federation of June 13, 2017 N 486n.

An individual program for the rehabilitation or habilitation of a disabled person (hereinafter referred to as IPRH) determines the list, content, scope, timing and level of rehabilitation measures to be carried out.

IPRH due to coronary artery disease includes in whole or in part a basic rehabilitation program (a guaranteed list of rehabilitation measures, technical means provided to a disabled person free of charge at the expense of federal or regional budget funding); at the same time, it can be expanded at the expense of individual rehabilitation measures, which are paid by the disabled person himself or by other persons and organizations. When implementing the IPRH in IHD, a disabled person is a subject of rehabilitation, who agrees to the implementation of an individual rehabilitation program and actively participates in its implementation.

It is necessary to take into account the gender, age of the disabled person and the nosological form of the disease at the developing the section of medical rehabilitation of IPRH.

In most cases, the degree of clinical, functional, occupational, and socio-environmental disorders is higher in elderly patients. Accordingly, their rehabilitation potential is lower. Meanwhile, some radical rehabilitation measures, for example: an effective operation to replace the valves of the heart and main arteries, the installation of a pacemaker, the creation of a special workplace in production, special equipment of the living quarters, the provision of special vehicles can completely neutralize the pronounced limitations of a disabled person's life.

The section of vocational rehabilitation differs between disabled people who didn't have a professional activity and for disabled people with a profession.

The section of social rehabilitation includes special (by gender and age) and specialized (depending on the microsocial environment). In order to carry out objective control over the quality and effectiveness of rehabilitation, state rehabilitation standards are established.

The expert and rehabilitation diagnostics carried out for the compilation of the IPRH in IHD is aimed at determining the degree of restriction of various types of vital activity, determining the rehabilitation potential (rehabilitation possibilities) and the rehabilitation prognosis of a patient or disabled person. Based on this, the need for the main types of medical and social rehabilitation is revealed [1].

Currently, rehabilitation potential is understood as a complex of biological and socio-environmental factors of a person, which allow, to one degree or another, to realize his potential capabilities. The main task of medical and social rehabilitation is the implementation of measures for the most effective implementation of the patient's rehabilitation potential.

Physiological and psychological aspects that determine the rehabilitation capabilities of disabled people with IHD are closely interrelated. Physiological rehabilitation capabilities largely determine the psychological status of a disabled person; the opposite picture is somewhat less common. Often, physiological rehabilitation possibilities are in conflict with the psychological attitudes of a disabled person.

The rehabilitation possibilities for the restoration of individual organs and functional systems in ischemic heart disease are variable. Therefore, the use of the generalizing concept of rehabilitation potential with an accepted assessment of it as high, medium and low seems to be very conditional. 
Assessment of the rehabilitation potential in IHD is multifactorial:

- $\quad$ Assessment of the medical component: The severity of the existing functional disorders; the severity of the course of the disease (stage, frequency of exacerbations, etc.); psychophysiological changes; restrictions and the possibility of their replacement or compensation.

- Assessment of the educational component: General education level; results of psychophysiological testing (personality traits, learning abilities, etc.).

- Assessment of the professional component: Compliance of the existing (preserved) functional capabilities with the requirements for professional activity in accordance with professional knowledge and skills while maintaining the previous workplace, changing the workplace with the need to be competitive in the open labor market, changing the workplace to a specialized one; job change with part-time employment, etc.; compliance of the existing functionality with the requirements of the new professional activity (work in a new profession with preliminary training, retraining, etc.; assessment of the compliance of the workplace and the technological process with the existing functional impairments and limitations).

- Assessment of the social and environmental component: Social damage in the ability to orientate (the ability of an individual to correctly orient himself in his environment); damage to physical independence (i.e. the existence of a situation in which adaptation to a normal and effective existence is possible only through the use of aids, devices, adaptation, family or the help of others); damage to stability (the ability of an individual to move enough in his environment or in his environment); damage to the ability to spend their time in accordance with their gender, age and culture; damage to social integration (the ability of an individual to participate in social relations and be compensated in this way); damage to economic independence [2].

The rehabilitation potential of a patient with coronary artery disease based on determining the rehabilitation capabilities of a disabled person in relation to the main categories of life: the ability to self-service, movement, learning, work, communication, orienta- tion and control over their behavior. This creates an opportunity to assess the degree of severity (criteria) of limitation of the main types of vital activity and to determine the body's capabilities for the real restoration of specific types of vital activity. It should be noted that the limitations of life activity arising in IHD are diverse and interrelated. Therefore, the successful implementation of the rehabilitation capabilities of a disabled person in the process of rehabilitation measures in terms of restoring a certain type of life activity leads to an increase in rehabilitation capabilities related to other types of life activity. This dynamism must be predicted and taken into account when carrying out rehabilitation measures.

As a basis for determining rehabilitation capabilities, along with the generally accepted methods of functional expert diagnostics aimed at determining the severity of functional disorders, special testing methods should be used using functional diagnostic tests that simulate various types of loads in life. These methods of assessing the condition of patients with coronary heart disease include:

- Clinical and instrumental examination of the function of the cardiovascular system with dosed physical exertion, with loads that simulate various types and modes of life, various environmental conditions electrocardiography, ECG monitoring, cardiointervalography, echocardiography, spiroergometry and other methods of studying the function of the circulatory system at dosed in intensity and the duration of physical and mental stress, simulation of stress, modes and conditions typical for everyday and professional activities, extreme conditions of life with increased or decreased ambient temperature, etc.

- Establishing the possibilities of function restoration (electrocardiography, echocardiography, X-ray examination, the number of methods of contrast angiocardiography and computed tomography, nuclear magnetic resonance imaging, etc.)

- Special psychological testing, detection of motivation to work. Determination of recovery opportunities, learning ability, etc.

- Psychophysiological testing related to certain types of activities or specific professions. 
- $\quad$ Ergonomic studies that determine the ability of a disabled person to carry out their life activities, including in the settings and working conditions on the data of a comprehensive physiological examination (according to the data of spiroergometry and the levels of energy consumption of the disabled person in the process of specific types and conditions of life activity).

An integral assessment of the rehabilitation potential in relation to the restoration of specific types of vital activity should be generalized and reflecting their level on the basis of clear quantitative indicators. It seems appropriate for practical use to distinguish three levels that characterize the rehabilitation potential of a disabled person: high, medium and low.

High rehabilitative potential presupposes complete or almost complete restoration of a specific type of life activity in the process of carrying out rehabilitation measures.

The middle (average) habilitation potential reflects the possibility of partial restoration of a specific type of vital activity in the process of carrying out rehabilitation measures.

Low rehabilitation potential predetermines the possible absence or insignificant result during rehabilitation measures aimed at restoring a specific type of vital activity [5].

On the basis of a certain rehabilitation factor and an analysis of the factors influencing its implementation, a characteristic of the rehabilitation prognosis is formed. Thus, the rehabilitation prognosis represents the estimated probability of realizing the rehabilitation potential. It is advisable to determine the rehabilitation prognosis in relation to specific types (categories) of vital activity. Rehabilitation prognosis can be assessed as:

- Favorable - the possibility of complete restoration of the disturbed categories of vital activity.

- $\quad$ Relatively favorable - the possibility of partial restoration of the disturbed categories of vital activity while reducing the degree of their limitations.

- Undefined.

- Unfavorable - the impossibility of even partial restoration of the disturbed categories of vital activity and reducing the degree of their limitations.
Rehabilitation prognosis in IHD mainly depends on the nosological form and nature of the course of the disease, the severity of functional disorders and disabilities, rehabilitation potential, and the expected influence of external factors.

Patients at the young age with moderate functional impairments and preserved ability to work, the priority in assessing the rehabilitation prognosis is the possibility of implementing medical and vocational rehabilitation programs.

At the age upper than the able-bodied with pronounced functional disorders and loss the ability to work the priority in assessing the rehabilitation prognosis is the possibility of implementing medical and social rehabilitation programs.

Thus, based on the determination of the presence and severity of disabilities, the need for and ways to eliminate or compensate them in comparison with the possibilities of their implementation, a rehabilitation prognosis is formulated [4].

A favorable rehabilitation prognosis is considered if it is possible to fully realize the rehabilitation potential, taking into account all three sections of the IPRH.

A relatively favorable rehabilitation prognosis is the possibility of partial realization of the rehabilitation potential in the event of its probable full realization only in accordance with priority programs.

Undefined prognosis is the possibility of partial realization of the rehabilitation potential in cases of probable unsatisfactory effectiveness of measures for priority rehabilitation programs.

An unfavorable prognosis in cases of impossibility of realizing the rehabilitation potential.

To determine the need for people with disabilities in medical and social rehabilitation, it is necessary to introduce into the practical activities of institutions of medical and social expertise and rehabilitation of disabled people a strict uniform codification approved by federal regulations in accordance with the International Classification of Functioning, Disabilities and Health (ICF) [6].

Medical and social expertise provides for the simultaneous identification of the severity of violations of a specific type of life and determination of the level of rehabilitation potential of a dis- 
abled person. Determination of the level of rehabilitation potential is carried out taking into account the degree of limitations of a particular category of life. Based on the determination of the degree of restriction of the main categories of life and the possibilities of their recovery, a specific need for the main types of medical and social rehabilitation of people with disabilities due to IHD is established.

\section{Conclusion}

The basis of medical and social rehabilitation of people with disabilities due to IHD is a specific targeted IPRH. Evaluation of the results of the rehabilitation measures carried out presents significant difficulties and may include various aspects. First of all, the result is the achieved level of integration of the disabled person into society. It seems promising to assess the results of the implementation of the IPRH in the following way: full integration of the disabled person into society; partial integration; absence or low result of integration of a disabled person [5].

The implementation of the above measures within the framework of individual and complex programs of rehabilitation or habilitation should be carried out under systematic clinical and instrumental control at all stages of the state of the functions of organs and systems of disabled people with the possibility of timely correction of rehabilitation measures.

\section{Bibliography}

1. Zabolotnykh I I and Kantemirova RK. "Clinical and expert diagnostics of pathology of internal organs: a guide for doctors". SPb (2007): 155-161.

2. Zabolotnykh I I and Kantemirova RK. "Medico-social examination and rehabilitation in cardiology: a guide for doctors". SPb (2008): 78-96.

3. Iksanov KhV. "Disability, medical and social expertise and rehabilitation of disabled people due to coronary heart disease in the Republic of Tatarstan". Dissertation work for the degree of candidate of medical sciences, Moscow (2003): 103-116.

4. Iksanov KhV and Isaenko SI. "Features of medical and social rehabilitation of disabled people due to coronary heart disease". Materials of the scientific and practical conference on the issues of disability, medical and social examination and rehabilitation of disabled people in the Republic of Tatarstan "Development of the regional system of medical and social examination and rehabilitation of disabled people". MoscowKazan (2004): 146-148.

5. Iksanov KhV., et al. "Modern approaches to medical and social examination of disabled people due to diseases of the circulatory system". Teaching aid, Kazan, KSMA (2012): 19-34.

6. Iksanov KhV., et al. "Aspects of the International Classification of Functioning, Disabilities and Health (ICF) in the Development of Individual Rehabilitation Programs". Modern Medicine 2 (2016): 63-65.

7. Puzin SN., et al. "Methodology and practice of development of medical and social expertise and rehabilitation of disabled people as a regional social system". Tonchu (2008): 67-82.

\section{Volume 5 Issue 8 August 2021}

(C) All rights are reserved by Bodrova Reseda A., et al. 